Glasgow Caledonian

University

University for the Common Good

\title{
Population impact of direct-acting antiviral treatment on new presentations of hepatitis C-related decompensated cirrhosis: a national record-linkage study
}

Hutchinson, Sharon J.; Valerio, Heather; McDonald, Scott A.; Yeung, Alan; Pollock, Kevin ; Smith, Shanley; Barclay, Stephen; Dillon, John F.; Fox, Raymond; Bramley, Peter; Fraser, Andrew; Kennedy, Nicholas; Gunson, Rory N.; Templeton, Kate; Innes, Hamish; McLeod, Allan; Weir, Amanda; Hayes, Peter C.; Goldberg, David

Published in:

Gut

DOI:

10.1136/gutjnl-2019-320007

Publication date:

2020

Document Version

Author accepted manuscript

Link to publication in ResearchOnline

Citation for published version (Harvard):

Hutchinson, SJ, Valerio, H, McDonald, SA, Yeung, A, Pollock, K, Smith, S, Barclay, S, Dillon, JF, Fox, R, Bramley, P, Fraser, A, Kennedy, N, Gunson, RN, Templeton, K, Innes, H, McLeod, A, Weir, A, Hayes, PC \& Goldberg, D 2020, 'Population impact of direct-acting antiviral treatment on new presentations of hepatitis Crelated decompensated cirrhosis: a national record-linkage study', Gut, vol. 69, no. 12, pp. 2223-2231.

https://doi.org/10.1136/gutjnl-2019-320007

Copyright and moral rights for the publications made accessible in the public portal are retained by the authors and/or other copyright owners and it is a condition of accessing publications that users recognise and abide by the legal requirements associated with these rights.

Take down policy

If you believe that this document breaches copyright please view our takedown policy at https://edshare.gcu.ac.uk/id/eprint/5179 for details of how to contact us. 


\section{Population impact of direct-acting antiviral treatment on new presentations of hepatitis C-related decompensated cirrhosis: a national record-linkage study}

Sharon J Hutchinson ${ }^{1,2}$, Heather Valerio ${ }^{1,2}$, Scott A McDonald ${ }^{1,2}$, Alan Yeung ${ }^{1,2}$, Kevin G Pollock ${ }^{1,2}$, Shanley Smith ${ }^{1,2}$, Stephen T Barclay ${ }^{1,3}$, John F Dillon ${ }^{4}$, Ray Fox ${ }^{5}$, Peter Bramley ${ }^{6}$, Andrew Fraser ${ }^{7,8}$, Nick Kennedy ${ }^{9}$, Rory Gunson ${ }^{10}$, Kate Templeton ${ }^{11}$, Hamish Innes ${ }^{1,2}$, Allan McLeod ${ }^{2}$, Amanda Weir ${ }^{2}$, Peter C Hayes ${ }^{12}$, David J Goldberg ${ }^{1,2}$

${ }^{1}$ School of Health and Life Sciences, Glasgow Caledonian University, Glasgow, UK;

${ }^{2}$ Health Protection Scotland, Glasgow, UK;

${ }^{3}$ Glasgow Royal Infirmary, Glasgow, UK;

${ }^{4}$ Ninewells Hospital and Medical School, Dundee, UK;

${ }^{5}$ The Brownlee Centre, Glasgow, UK;

${ }^{6}$ Stirling Royal Infirmary, Stirling, UK;

${ }^{7}$ Aberdeen Royal Infirmary, Aberdeen, UK;

${ }^{8}$ Queen Elizabeth University Hospital, Glasgow, UK;

${ }^{9}$ University Hospital Monklands, Lanarkshire, UK;

${ }^{10}$ West of Scotland Specialist Virology Centre, Glasgow Royal Infirmary, Glasgow, UK;

${ }^{11}$ East of Scotland Specialist Virology Centre, Royal Infirmary of Edinburgh, Edinburgh, UK;

${ }^{12}$ Royal Infirmary Edinburgh, Edinburgh, UK.

Correspondence to:

Professor Sharon Hutchinson, School of Health and Life Sciences, Glasgow Caledonian University, Cowcaddens Road, Glasgow G4 0BA, UK. Email: sharon.hutchinson2@nhs.net; Tel: +44(0)141 273 1949.

Word count: 4,196; abstract 253

Key words: Hepatitis C, cirrhosis, antiviral therapy, surveillance, linkage analysis

Abbreviations:

CC Compensated cirrhosis

DAA Direct-acting antiviral

DC Decompensated cirrhosis

HCC Hepatocellular carcinoma

HCV Hepatitis C virus

HPS Health Protection Scotland

ICD International Classification of Diseases

NHS National Health Service

PCR Polymerase chain reaction

PWID People who injects drugs

RNA Ribonucleic acid

RR Rate ratio

SVR Sustained viral response

WHO World Health Organization 


\section{Summary}

Objective Population-based studies demonstrating the clinical impact of interferon-free direct-acting antiviral (DAA) therapies are lacking. We examined the impact of the introduction of DAAs on HCVrelated decompensated cirrhosis (DC) through analysis of population-based data from Scotland.

Design Through analysis of national surveillance data (involving linkage of HCV diagnosis and clinical databases to hospital and deaths registers), we determined i) the scale-up in the number of patients treated and achieving a sustained viral response (SVR), and ii) the change in the trend of new presentations with HCV-related DC, with the introduction of DAAs.

Results Approximately 11000 patients had been treated in Scotland over the eight-year period 2010/11 to 2017/18. The scale-up in the number of patients achieving SVR between the pre-DAA and DAA eras was 2-3-fold overall and 5.9-fold among those with compensated cirrhosis (the group at immediate risk of developing DC). In the pre-DAA era, the annual number of HCV-related DC presentations increased 4.6-fold between 2000 (30) and 2014 (142). In the DAA era, presentations decreased by 51\% to 69 in 2018 (and by 67\% among those with chronic infection at presentation), representing a significant change in trend (rate ratio $0 \cdot 88$, 95\% CI 0.85 to 0.90 ). With the introduction of DAAs, an estimated 330 DC cases had been averted during 2015-18.

Conclusions National scale-up in interferon-free DAA treatment is associated with the rapid downturn in presentations of HCV-related DC at the population-level. Major progress in averting HCV-related DC in the short-term is feasible, and thus other countries should strive to achieve the same. 


\section{Significance of this study}

\section{What is already known on this subject?}

Decompensated cirrhosis (DC) is the major sequelae of chronic hepatitis C virus (HCV) infection, accounting for the majority (65\%) of the 400,000 HCV related-deaths occurring worldwide each year.

$>$ Cohort studies have demonstrated that exposure to new direct-acting antivirals (DAAs) for chronic HCV to be associated with a decrease in all-cause mortality and hepatocellular carcinoma, but findings were inconclusive for DC.

> Studies showing the population-level impact of DAAs on chronic HCV-related sequelae are lacking.

\section{What are the new findings?}

$>$ At a population-level, new presentations of chronic HCV-related DC fell by $67 \%$ within the first four years of DAAs, contemporaneous with national scale-up of these new therapies in Scotland.

$>$ For newly diagnosed compensated cirrhotic patients, the risk of presenting with DC was halved in the DAA, compared to pre-DAA, era.

\section{Implications of all the available evidence}

Countries should aim to make major progress in averting HCV-related DC, ahead of WHO global targets on elimination of viral hepatitis in 2030. 


\section{Introduction}

Worldwide, 71 million people are estimated to be living with the hepatitis C virus (HCV). ${ }^{1}$ In the absence of therapeutic intervention, chronic HCV infection can lead to compensated cirrhosis (CC), a generally asymptomatic state until onset of severe sequelae, notably decompensated cirrhosis (DC). ${ }^{2}$ The latter is life-threatening, requiring liver transplantation to avoid an otherwise $70 \%$ mortality risk within five years. ${ }^{3}$ Cirrhosis thus accounts for the majority (65\%) of the 400,000 HCV-related deaths worldwide each year. ${ }^{1}$

The new generation of direct-acting antiviral (DAA) drugs for HCV are extraordinarily effective, well-tolerated and of short (8-12 week) duration. ${ }^{4}$ Carefully conducted clinical trials and real-world studies have demonstrated the ability of DAAs to clear HCV from the host, resulting in a sustained viral response (SVR) in excess of $90 \%$ of patients' treated. ${ }^{5,6}$ In the pre-DAA era, SVR achievement was associated with approximately $80 \%$ reduction in risk for severe liver outcomes. ${ }^{7,8}$ Treatment with DAA therapy has therefore the potential to profoundly change the course of rising trends in HCV-related disease burden. ${ }^{9,10}$ However, the clinical utility of a DAA-induced SVR has been disputed by authors of a Cochrane review who have advocated that the real-world impact of DAA therapy on HCV-related liver morbidity remains unproven. ${ }^{11,12}$ Data from population-based studies are thus in demand to corroborate the expected clinical benefit from these drugs. ${ }^{13,14,15}$

Due to the high cost of DAA drugs and large number of people infected, Scotland, like many other countries, initially prioritised patients with advanced liver fibrosis; in addition, an ambitious government target was set, informed by mathematical modelling, to reduce the number of new presentations for HCV-related DC nationally by $75 \%$ within five years. ${ }^{9,16}$ Scotland - through establishment of national surveillance of $\mathrm{HCV}$, unparalleled elsewhere, covering diagnoses, treatment and disease - is well placed to assess the population impact of DAAs on hepatic sequelae. ${ }^{17}$ Herein, we evaluated the early impact of Scotland's strategy, in relation to the introduction of interferon-free DAA therapy, on i) the scale-up of patients treated for HCV and achieving SVR (particularly among those with CC, the group at immediate risk of developing DC), and ii) new presentations with HCVrelated DC at the population level.

\section{Methods}

\section{Study design}

Through analysis of national surveillance data, we examined changes over time in the uptake and impact of HCV treatment in Scotland. As previous modelling work had indicated that a three-fold increase in HCV treatment among those with advanced liver fibrosis could lead to immediate impact in preventing HCV-related DC, ${ }^{9}$ we gauged the scale-up in the number of patients (overall and among those with CC) initiated on therapy and achieving SVR in the interferon-free DAA era (simplified to $D A A$ era hereafter) compared to the pre-DAA era. In the context of this scale-up, we examined the temporal trend in new presentations for HCV-related DC in Scotland and assessed whether there had been any change in this trend - and, related to that, estimated the number of cases averted - since the introduction of DAAs. To corroborate this population-level analysis, we also undertook an individuallevel analysis which retrospectively followed up a national cohort with HCV-related cirrhosis to determine whether the risk of DC differed for those diagnosed with cirrhosis in the DAA era compared to prior to then. Supplementary file 1 includes an overview on the databases, record-linkage and analysis involved.

\section{Data Sources}

Scale-up in patients treated for HCV and achieving SVR

Health Protection Scotland (HPS) collates national surveillance data on patients initiated onto HCV therapy for monitoring against government targets (in place since 2008) ${ }^{18}$. This is in the form of monthly reporting by NHS boards on aggregate numbers, in addition to individual-level data that are downloaded annually from standardised Clinical Databases installed in (17/18) specialist HCV 
treatment centres across Scotland. ${ }^{7}$ Data held on the Clinical database includes HCV genotype, date of diagnosis with cirrhosis or hepatocellular carcinoma (HCC), date of HCV treatment initiation and completion, treatment regimen, and response to therapy. A diagnosis of cirrhosis is made based on standard clinical practice through a combination of liver biopsy, transient elastography, abdominal ultrasound, clinical examination and routine liver function tests. ${ }^{19,20}$ Data to the end of March 2018 were used, enabling four (financial) years of treatment data pre and post introduction of DAAs, for analysis.

New presentations for HCV-related DC

National data on people who have ever been diagnosed with anti-HCV and their subsequent PCR results are also held at HPS, through collation of administrative data from the four laboratories undertaking all confirmation HCV antibody and PCR testing in Scotland. ${ }^{21}$ Through record-linkage of the Diagnosis database (to the end of 2018, involving 43,080 persons with anti-HCV) to morbidity and mortality databases, the number of people diagnosed with HCV and presenting to hospital with, or otherwise died from, DC in Scotland was determined. ${ }^{1822}$ The hospital data, held by the Information Services Division (ISD), involves records of all general and acute inpatient/day-case hospital discharges in Scotland; discharge diagnoses were coded using the International Classification of Diseases (ICD)-9 during 1989-1995 and ICD-10 thereafter. Death registration data were provided by National Records of Scotland; underlying and contributing causes of death were coded using ICD9 during 1989-1999 and ICD-10 thereafter. Linkage of the Diagnosis database to the Scottish HCV Test (containing PCR and core-antigen test data since 1999) ${ }^{23}$ and Clinical databases (containing SVR status) was also undertaken to ascertain chronic HCV status at the time of DC presentation (described below). Further, linkage of the HCV Clinical database to morbidity and mortality databases afforded an opportunity to in addition examine presentations with HCV-related DC among a national cohort of people attending specialist HCV centres. The linkage exercise, approved by the Scottish Public Benefit and Privacy Panel, was performed by ISD in May 2019. All databases involved hold the Community Health Index number (a unique identifier allocated to each patient in NHS Scotland), which was used to link the Diagnosis database to, and retrieve records from, other databases (as described elsewhere), ${ }^{3,7,19}$

\section{Outcome Measures}

Scale-up in patients treated for HCV and achieving SVR

The number, and characteristics, of patients initiated onto HCV therapy in Scotland in each financial year from April 2010 to March 2018 was ascertained through the Clinical database for 17/18 specialist centres, supplemented with aggregate-level data reported to HPS for the remaining centre. We examined the proportion of patients achieving SVR, defined as testing PCR negative for viral RNA for a period of 12 or 24 weeks following completion of therapy, according to clinical guidelines We also estimated the number of people achieving SVR according to each financial year of treatment initiation, by combining the number of SVR cases where known with an estimate of SVR cases for those treatment initiations where SVR response was not known; for the latter, we applied the SVR rates (for those with a known response) to those who had completed their treatment course.

New presentations for HCV-related DC: population-level analysis

Presentations of HCV-related DC were defined as first-time admissions for DC among persons who had ever been diagnosed with chronic HCV infection and identified through the linkage of the Diagnosis database to the hospital data up to the end of 2018. Admission for DC referred to a hospitalisation with any of the following recorded in either the primary and/or secondary diagnostic fields: ascites (ICD-10: R18; ICD-9 789.5); bleeding oesophageal varices (ICD-10: I85·0, I98·3; ICD9 456.0); chronic or alcoholic hepatic failure, including hepatic encephalopathy (ICD-10: K70.4, K72.1, K72.9; ICD-9: 572.2, 572.8); and hepatorenal syndrome (ICD-10: K76.7; ICD-9: 572.4). These diagnosis codes were selected based on a previous validation exercise and have been applied in studies from Scotland and elsewhere. ${ }^{22,24}$ To reduce potential bias due to DC arising from a different aetiology, patients whose first DC admission occurred more than one year preceding their HCV diagnosis were excluded. Persons diagnosed with a history of chronic HCV was deduced through the PCR test data captured on the HCV diagnosis and test databases; those who had spontaneously 
resolved their infection (i.e. PCR negative at time of their HCV diagnosis, with no subsequent record to indicate PCR positive) or whose PCR status was not known were excluded from analysis, whilst those who had cleared infection from HCV treatment were retained. In sensitivity analyses, we considered two broader definitions of HCV-related DC which additionally included either (a) those individuals who had not presented to hospital with DC but had otherwise died from DC (using the same set of ICD codes as above in relation to primary or secondary cause of death), or (b) those individuals diagnosed with anti-HCV but whose PCR status was not known.

New presentations for HCV-related DC: individual-level analysis

We also examined the risk of presentation with HCV-related DC (as described above) in the DAA compared to pre-DAA era at an individual-level through analysis of linked (hospital and deaths) data for a national cohort of patients with CC attending specialist HCV centres. The study population here was confined to patients who were recorded on the Clinical database as having chronic HCV and had been diagnosed with cirrhosis since January 2011; those with evidence of decompensation or HCC at baseline were excluded. We confined the pre-DAA era to a period (since 2011) when transient elastography (FibroScan ${ }^{\circledR}$ ) was routinely used to diagnose cirrhosis, thereby minimising any bias arising through adoption of different modes of diagnosis across the study period. ${ }^{25}$

\section{Statistical Analysis}

Population-level analysis

Segmented Poisson regression was used to assess the change in presentations of HCV-related DC nationally, following the introduction of DAAs. ${ }^{26}$ For the purpose of the modelling, presentations of HCV-related DC were split into six-monthly intervals, and categorised as pre-DAA (January 2000 to December 2014) and DAA (January 2015 to December 2018) eras. A change point at the end of 2014 was used in the model, allowing time for treatment initiation and completion (involving up to 24 weeks of therapy initially) in the period immediately after the licencing of the first DAA (Sofosbuvir) in Scotland during June 2014. ${ }^{27}$ The segmented regression model estimates the temporal trend in presentations of HCV-related DC in both the pre-DAA and DAA eras, as well as the change in trend between the two periods. Results are presented as rate ratios (RR) with 95\% CIs and associated pvalues. Models were performed for all patients, and stratified across a range of relevant factors (at DC presentation if not otherwise stated): sex, age $(<50,50+$ years), DC diagnosis category (defined hierarchically in order of hepatic failure, ascites and bleeding varices based on previous work $)^{3}$, presence of HCC (based on ICD-10 C22.0), chronic HCV status (based on linked HCV laboratory test and clinical data, as described above), and alcohol-related admission history (based on ICD diagnosis codes, as described elsewhere, ${ }^{24}$ in any hospital record up to and including the DC admission). We also examined the extent to which HCV was coded on the DC hospital record (based on ICD-10 B17.1, B18.2), thereby providing insight as to the extent to which HCV-related DC would have been captured based solely on these data. Finally, we estimated the cumulative number of HCV-related cases averted with the introduction of DAAs, by subtracting the observed number from the counterfactual number - the latter predicted by the model based on the temporal trend before the introduction of DAAs - for the period 2015-18.

\section{Individual-level analysis}

Multifactorial Cox regression was used to estimate the risk of presentation with HCV-related DC among the national cohort of compensated cirrhotic patients, comparing those diagnosed with cirrhosis in a period preceding the introduction of new DAAs (January 2011 to May 2014) versus those diagnosed after (June 2014 to March 2018). Follow-up for each patient began at the date of cirrhosis diagnosis and ended at the earliest of the date of DC presentation, death, migration or administrative right-censoring date ( $31^{\text {st }}$ March 2018). Covariates included in the regression were: sex, age group at cirrhosis diagnosis, risk group for HCV acquisition (person who injects drugs (PWID), non-PWID), alcohol consumption history ( $>50$ units per week, $\leq 50$ units per week/not known), genotype (1,3, other/not known), antiviral treatment experience prior to cirrhosis diagnosis and Child-Pugh class (A, B). For the latter, the most recent laboratory test result (for bilirubin, albumin, and prothrombin) within 120 days of cirrhosis diagnosis was used. ${ }^{25}$ Multiple imputation 
using chained equations was performed using the $\mathrm{R}$ package mice to impute missing data on risk group and laboratory measurements needed to compute Child-Pugh class. ${ }^{28,29}$

Statistical analyses were conducted using R (version 3.5.1) and Stata (version 14).

\section{Results}

\section{Scale-up in patients treated for HCV and achieving SVR}

Approximately 11000 patients had been initiated on HCV therapy in Scotland during the eight-year period 2010/11 to 2017/18 (Table 1), involving a 1.7-fold rise in initiates in the DAA compared to the pre-DAA period (Figure 1a). Among patients diagnosed with CC, a greater scale-up of HCV treatment was observed, involving a 3.2-fold rise in the DAA compared to pre-DAA era (Figure 1b). The proportion of treatment initiations involving an interferon-free DAA regimen increased from $27 \%$ in $2014 / 15$ to $72 \%$ in $2015 / 16$, reaching $98 \%$ by $2017 / 18$ (Table 1 ).

Among 9237 patients initiated on HCV treatment during April 2010 to September 2018, 71\% had achieved SVR (supplementary file 2). Confined to those where SVR status was known (87\% of those treated), the proportion of treatment initiates achieving SVR rose from $76 \%$ in the pre-DAA era to $94 \%$ in the DAA era. Among those with CC, this rise was more pronounced: increasing from $59 \%$ in the pre-DAA era to $92 \%$ in the DAA era. The scale-up in the estimated number of patients achieving SVR between the pre-DAA and DAA eras was 2·3-fold overall and 5.9-fold among those with CC (Figure 1).

New presentations for HCV-related DC: Population-level analysis

In the pre-DAA era, the annual number of presentations with HCV-related DC increased from 31 in 2000 to 142 in 2014 (Figure 2). Since then (in the DAA era), the annual number had fallen to 69 in 2018 (i.e. the lowest number for over a decade); this represents a 51\% reduction among those with a history of chronic HCV. Considering only those with chronic HCV at the time of DC presentation, the number of DC presentations reduced by 67\% between 2014 (127) and 2018 (42).

The characteristics of patients presenting with HCV-related DC across the two eras were similar (Table 2a), except those presenting in the DAA era were older compared to the pre-DAA era (53\% and $36 \%$ aged $\geq 50$ years, respectively) consistent with an ageing cohort as reported elsewhere (and in the individual-level analysis, supplementary file 4$).{ }^{18}$ In addition, a higher proportion had already cleared HCV from therapy among those presenting in the DAA compared to pre-DAA era (25\% and $6 \%$, respectively). The minority of DC presentations had HCV coded on their hospital record (39\% in the pre-DAA and $42 \%$ in the DAA era).

The number of HCV-related DC presentations was estimated to have increased 4\% per six-month period in the pre-DAA era, and thereafter decreased $8 \%$ per six-months in the DAA era (Figure 2); this represents a significant change in trend for the DAA compared to pre-DAA era (RR $0 \cdot 88,95 \%$ CI 0.85-0.90) (Table 2b). In sensitivity analysis, results were unchanged considering two broader definitions for HCV-related DC (supplementary file 3). An increasing trend in HCV-related DC was observed across all strata considered in the pre-DAA era (Table 2b); that trend changed significantly in the DAA era across most strata (according to sex, age groups, alcohol admission history and DC diagnosis categories), with the exception that no significant change was found for those who had HCV coded on the DC hospital record and those who had cleared HCV prior to DC presentation. Without the introduction of DAAs, the predicted annual number of HCV-related DC presentations was estimated to have increased to 208 by 2018, which translates to an estimated 330 cases averted with the introduction of DAAs during 2015-18 (Figure 2).

New presentations for HCV-related DC: Individual-level analysis

Among the national compensated cirrhotic cohort $(n=2249)$, 55\% were newly diagnosed in the DAA era, $74 \%$ were male, $38 \%$ aged $\geq 50$ years, $64 \%$ PWID, $33 \%$ had a history of heavy alcohol use, $46 \%$ were genotype 3, 3\% had Child-Pugh B, and 17\% were treatment experienced (Table 3). These 
characteristics were similar between those diagnosed with cirrhosis in the pre-DAA and DAA eras, with the exception of prior treatment experience (25\% and $10 \%$, respectively) (supplementary file 4). Compared to those diagnosed with cirrhosis in the pre-DAA era, patients diagnosed with cirrhosis in the DAA era had a significantly reduced risk of presenting with HCV-related DC (hazard ratio (HR) $0 \cdot 55,95 \%$ CI 0.40-0.76), after adjusting for covariates (Table 3).

\section{Discussion}

These findings provide the first evidence of a major population impact of DAAs in terms of averting HCV-related DC at a country-level. In line with the initial national strategy on DAAs in Scotland, scale-up of treatment was greatest for those with CC (the group at immediate risk of DC), and translated to a near six-fold rise in the estimated number of such patients clearing the virus and achieving SVR between the pre-DAA and DAA eras. This represents a level of change in DAA treatment uptake and clearance of infection that previous modelling work had predicted would yield an immediate reduction in the incident number of HCV-related DC. ${ }^{7}$ A halving in new presentations of DC among those with a history of chronic HCV has been observed within the first four years of DAAs; an even greater reduction of $67 \%$ was observed for those with chronic HCV at the time of presentation (i.e. excluding those who had received HCV therapy and were clear of their infection prior to presentation of DC). Our data provide compelling evidence to policymakers and practitioners that, in the context of an evidenced-based national plan, DAA treatment can rapidly revert an increasing tide of HCV-related DC.

The above differential finding - vis-à-vis a 51\% reduction among those with chronic HCV either currently or in the past versus $67 \%$ reduction among those with chronic HCV currently - was anticipated. Previous work has shown that heavy alcohol use, as well as other health risk behaviours known to accelerate liver disease progression, are prevalent in HCV-infected populations both in Scotland and elsewhere, and may compromise the clinical benefit of DAAs. ${ }^{20,24,30}$ New DC presentations among those who had cleared HCV from therapy almost doubled from 15 in 2014 to 27 in 2018; 64\% of the latter had an alcohol-related admission history. This rise reflects the considerable expansion of the compensated cirrhotic SVR population since the introduction of DAAs and residual risk of liver disease progression associated with alcohol and other risk behaviours. Overall, we found a similar significant change in the trend of DC presentations between the pre-DAA and DAA eras for both those with and without an alcohol history (captured through alcohol-related hospital episodes). Further, our individual-level analysis demonstrated a $45 \%$ reduced risk of presentation with DC for patients diagnosed with CC in the DAA era compared to those diagnosed in the pre-DAA era, adjusting for alcohol consumption history and other factors. Whilst our findings reinforce the need to address health risk behaviours in HCV infected and resolved populations (particular those with cirrhosis and at continued risk of liver failure and cancer), they also demonstrate that averting HCVrelated DC is possible even within sub-populations with a history of heavy alcohol use.

Our results directly address the perspective that the real-world impact of DAA therapy remains unproven at the population level. ${ }^{11,12}$ Previously, data from Liver Transplant Registries in the United States and Europe have provided evidence of the early impact of DAAs, commensurate with our findings. ${ }^{31,32}$ Within the first two years of DAAs in Australia (via initially their compassionate access programme), a $21 \%$ reduction in presentations of HCV-related DC was observed at the state level of New South Wales among individuals diagnosed with anti-HCV; thus, a limitation was that individuals who had spontaneously resolved their HCV (over 25\% of all diagnoses) were included in this trends analysis (as data on chronic infection and therapy were lacking), and may explain the lower rate of decline in the DAA era (3\% per 6 months) compared to that observed for Scotland (8\% per 6 months). ${ }^{33}$ Individual-level analysis of a large multi-centre cohort study from France demonstrated that patients treated with DAAs, compared to those untreated, was associated with a decrease in allcause mortality and HCC but failed to find an association with DC (adjusted HR 1.14, 0.57-2.27; $\mathrm{p}=0.72){ }^{34}$ The lack of an association with DC could be due to limited statistical power, as only 106 DC events were observed and their analysis relied on adjustment for 19 covariates in the multivariable model to explain the otherwise higher crude incidence of DC among those treated compared to 
untreated. ${ }^{34}$ Both the population-level and individual-level analysis presented here from Scotland involved greater numbers of DC events (1706 and 219, respectively) to draw conclusions. Further, our individual-level analysis by design involved groups (i.e. those newly diagnosed with CC in the preDAA and DAA eras) similar in nature and less prone to confounding issues as is evident with a treated to untreated comparison.

The major strength of our study, unique to Scotland, is the availability of national data monitoring HCV diagnosis, treatment and disease, established in advance of the DAA era. ${ }^{17}$ Whilst many countries have adopted a similar DAA strategy to Scotland (vis-à-vis initially prioritising patients with advanced fibrosis), few have developed surveillance that can demonstrate populationlevel impact of treatment, despite calls from WHO to invest in systems to monitor progress towards elimination of viral hepatitis. ${ }^{1,35}$ Without such surveillance, countries lack knowledge to inform the extent and nature of additional effort needed to prevent severe consequences of HCV.

Our study is not without limitations. First, we rely on the ICD coding of DC and this could be subject to possible misclassification error. Our analysis reassuringly yielded a similar trend when stratified according to the major clinical indicators of DC (i.e. hepatic failure, ascites and bleeding varices). Importantly, we did not rely on the ICD coding of HCV on the hospital or death record as is well known to under-estimate HCV-related outcomes. ${ }^{36,37}$ Due to the severe nature of DC, almost all cases present to hospital, at which point HCV testing is (or has already been) performed, and so few presentations should be missed through our record-linkage approach. Second, we may have overestimated the number of HCV-related DC cases averted with DAAs if the assumed counterfactual for the period 2015-18 had not kept pace with the increasing trend for the fifteen-year period up to that point, but our assumption is in keeping with previous modelling work for Scotland which forecasted such a trajectory to at least 2020. ${ }^{2}$ Third, whilst a favourable change in HCV-related DC presentations was observed for those with a history of excess drinking, we cannot draw conclusions on whether this holds for those who continue to drink to excess. Fourth, other comorbidities influencing liver disease progression such as HIV and obesity, which are less prevalent in the Scottish HCV population ${ }^{20,22}$, would be worthy of study in other settings where these feature to a greater extent. Fifth, we confined our investigation to DC. Justification for this was to provide a thorough analysis for the leading cause of HCV-related mortality and the lack of an association between DAAs and DC by Carrat et al. meant a closer examination was warranted. ${ }^{34}$ Further, whilst a decline in new presentations of HCV-related DC was expected within the first few years of DAAs, population-level change in the incident number of HCC cases and liver-related deaths may take longer to accrue (as these clinical endpoints stem not just from compensated cirrhosis, but also from more advanced liver disease) and thus longer followup, than that considered here, would be beneficial to measure impact on these other outcomes; indeed, preliminary analysis shows the downturn in new presentations of HCV-related HCC and HCV-related liver deaths in Scotland occurred, subsequent to the fall in HCV-related DC, towards the end of the follow-up period in 2017 and 2018, respectively (supplementary file 5). Sixth, whilst we have examined the scale-up of DAAs and associated impact for a predominantly injecting drug use-related HCV epidemic, we have not considered those currently injecting separate from those who injected in the past. Data from a national survey in Scotland however show that appreciable scale-up of DAAs among the population continuing to inject drugs did not occur until towards the end of the study period considered here (during 2017-2018) and thus is the subject of an ongoing study to determine whether treatment leads to prevention benefit. ${ }^{38,39}$

In summary, this study provides evidence at the population-level of a major decline in presentations of HCV-related DC associated with the introduction of interferon-free DAAs. Compared to the pre-DAA era, patients diagnosed with cirrhosis in the DAA era have a significant reduced risk of progressing to DC. Our data underpin the new Scottish Government strategy and commitment to eliminate HCV by the end of 2024, involving reducing new presentations of DC among those with chronic HCV to less than ten per year, as well as reducing the prevalence of infection to below $0.1 \% .{ }^{40,41}$ Whilst it remains to be seen as to whether we can eliminate HCV in Scotland within the next five years, we have nevertheless demonstrated that major progress in averting DC in the short-term is feasible, and so other countries should strive to achieve the same.

\section{Contributors}


SJH and DJG conceived and designed the study. SJH, HV, AY, SS, STB, JFD, RF, PB, AF, NK, RG, $\mathrm{KT}, \mathrm{HI}, \mathrm{AM}, \mathrm{AW}, \mathrm{PCH}$ and DJG contributed to the implementation of national surveillance of HCV infection in Scotland. SJH, HV, SAM, AY and SS contributed to the data analysis and generation of result tables and figures. All authors interpreted the findings. SJH, with support from HV, SAM and KGP, drafted the manuscript and all remaining authors contributed to critical review and development of the final manuscript.

\section{Declaration of interests}

Professor Hutchinson reports grants from Health Protection Scotland, during the conduct of the study; personal fees from Gilead, outside the submitted work. Dr. Barclay reports grants, personal fees and other from Gilead; grants, personal fees and other from Abbvie; all outside the submitted work. Dr. Fox reports personal fees from Gilead, outside the submitted work. Dr. Templeton reports grants from GenMark, grants from Cepheid, grants from Qiagen, other from SpeedX, all outside the submitted work. Professor Hayes reports personal fees from Gilead, personal fees from Abbvie, personal fees from BMS, personal fees from Jannsen, personal fees from Roche, all outside the submitted work. Dr. Kennedy reports personal fees from Gilead, personal fees from AbbVie, all outside the submitted work. Professor Dillon reports grants and personal fees from Gilead, grants and personal fees from BMS, grants and personal fees from MSD, grants and personal fees from Janssen, grants and personal fees from Roche, grants and personal fees from Abbvie, all outside the submitted work. All remaining authors declare no competing interests.

\section{Acknowledgments}

This work was supported by funding from Health Protection Scotland. We acknowledge the contributions of all staff at HCV testing laboratories and HCV treatment centres for their roles in the implementation of the national HCV surveillance programme in Scotland. We would also like to thank the Information Services Division, NHS National Services Scotland, for their role in undertaking the annual record-linkage of national surveillance databases. Additionally, we express gratitude to Scottish Government, the National Blood Borne Virus Coordinators Network, the National Viral Hepatitis Clinical Leads Network, the National Monitoring Assurance and Research Group and the Scottish Government's Hepatitis C Treatment and Therapies Group for their input to, and ongoing support of, the HCV surveillance programme in Scotland. We would like to acknowledge the key role of Hepatitis Scotland and the Hepatitis C Trust, as patient representatives to the aforementioned national advisory groups, for their input to the formation and continual review of the HCV surveillance data reported here.

\section{Indirect Patient and Public Involvement}

We did not directly include PPI in this study, but the public health surveillance data and associated outcome measures used in this study were developed with PPI involvement and are regularly reviewed by national advisory groups that include patient representatives.

\section{References}

1. WHO. Global hepatitis report, 2017. April, 2017. www.who.int/hepatitis/publications/globalhepatitis-report2017/en/ (accessed September 20, 2019)

2. Hutchinson SJ, Bird SM, Goldberg DJ. Modeling the current and future disease burden of hepatitis C among injection drug users in Scotland. Hepatology 2005; 42: 711-23.

3. McDonald SA, Innes HA, Aspinall E, et al. Prognosis of 1169 hepatitis C chronically infected patients with decompensated cirrhosis in the pre direct-acting antiviral era. $J$ Viral Hepat 2017; 24: 295-303.

4. WHO. Guidelines for the care and treatment of persons diagnosed with chronic hepatitis $\mathrm{C}$ virus infection. July, 2018. www.who.int/hepatitis/publications/hepatitis-c-guidelines-2018/en/ (accessed September 20, 2019) 
5. Fathi H, Clark A, Hill NR, Dusheiko G. Effectiveness of current and future regimens for treating genotype 3 hepatitis C virus infection: a large-scale systematic review. BMC Infect Dis 2017; 17: 722.

6. Falade-Nwulia O, Suarez-Cuervo C, Nelson DR, Fried MW, Segal JB, Sulkowski MS. Oral Direct-Acting Agent Therapy for Hepatitis C Virus Infection: A Systematic Review. Ann Intern Med 2017; 166: 637-48.

7. Innes HA, McDonald SA, Dillon JF, et al. Toward a more complete understanding of the association between a hepatitis $\mathrm{C}$ sustained viral response and cause-specific outcomes. Hepatology 2015; 62: 355-64.

8. Simmons B, Saleem J, Heath K, Cooke GS, Hill A. Long-Term Treatment Outcomes of Patients Infected With Hepatitis C Virus: A Systematic Review and Meta-analysis of the Survival Benefit of Achieving a Sustained Virological Response. Clin Infect Dis 2015; 61: 730-40.

9. Innes H, Goldberg D, Dillon J, Hutchinson SJ. Strategies for the treatment of Hepatitis C in an era of interferon-free therapies: what public health outcomes do we value most? Gut 2015; 64: 1800 9.

10. European Union HCV Collaborators. Hepatitis $C$ virus prevalence and level of intervention required to achieve the WHO targets for elimination in the European Union by 2030: a modelling study. Lancet Gastroenterol Hepatol 2017; 2: 325-36.

11. Jakobsen JC, Nielsen EE, Feinberg J, et al. Direct-acting antivirals for chronic hepatitis C. Cochrane Database Syst Rev 2017; 9: CD012143.

12. Jakobsen JC, Nielsen EE, Koretz RL, Gluud C. Do direct acting antivirals cure chronic hepatitis C? BMJ 2018; 361: k1382.

13. Wiktor SZ, Scott JD. What is the impact of treatment for hepatitis C virus infection? Lancet 2017; 390: 107-9.

14. Lok AS, Chung RT, Vargas HE, Kim AY, Naggie S, Powderly WG. Comments on cochrane review on direct-acting antivirals for hepatitis C. Hepatology 2017; 66: 1016-9.

15. European Association for the Study of the Liver. Response to the Cochrane systematic review on DAA-based treatment of chronic hepatitis C. J Hepatol 2017; 67: 663-664.

16. Scottish Government. Hepatitis C Treatment and Therapies Group Report. December, 2015. www2.gov.scot/Resource/0050/00501921.pdf (accessed September 20, 2019)

17. Hutchinson SJ, Dillon JF, Fox R, et al. Expansion of HCV treatment access to people who have injected drugs through effective translation of research into public health policy: Scotland's experience. Int J Drug Policy 2015; 26: 1041-9.

18. McLeod A, Glancy M, Went A, et al. HPS Surveillance Report: Surveillance of hepatitis C testing, diagnosis and treatment in Scotland, 2019 update. Health Protection Scotland, July 2019. hpspubsrepo.blob.core.windows.net/hps-website/nss/2834/documents/1_hcv-testing-diagnosistreatment-scotland-2018.pdf (accessed September 20, 2019)

19. Innes H, Barclay ST, Hayes PC, et al. The risk of hepatocellular carcinoma in cirrhotic patients with hepatitis C and sustained viral response: Role of the treatment regimen. $J$ Hepatol 2018; 68: 646-54.

20. Innes HA, Hutchinson SJ, Barclay S, et al. Quantifying the fraction of cirrhosis attributable to alcohol among chronic hepatitis $C$ virus patients: implications for treatment cost-effectiveness. Hepatology 2013; 57: 451-60.

21. Shaw L, Taylor A, Roy KM, et al. Establishment of a database of diagnosed HCV-infected persons in Scotland. Commun Dis Public Health 2003; 6: 305-10.

22. McDonald SA, Hutchinson SJ, Bird SM, et al. Hospitalization of hepatitis C-diagnosed individuals in Scotland for decompensated cirrhosis: a population-based record-linkage study. Eur J Gastroenterol Hepatol 2010; 22: 49-57.

23. McLeod A, Weir A, Aitken C, Gunson R, Templeton K, Molyneaux P, McIntyre P, McDonald S, Goldberg D, Hutchinson S. Rise in testing and diagnosis associated with Scotland's Action Plan on Hepatitis C and introduction of dried blood spot testing. J Epidemiol Community Health 2014; 68: $1182-8$.

24. Alavi M, Janjua NZ, Chong M, et al. The contribution of alcohol use disorder to decompensated cirrhosis among people with hepatitis C: An international study. J Hepatol 2018; 68: 393-401. 
25. McDonald SA, Barclay ST, Hutchinson SJ, et al. Uptake of endoscopic screening for gastroesophageal varices and factors associated with variceal bleeding in patients with chronic hepatitis C infection and compensated cirrhosis, 2005-2016: a national database linkage study. Aliment Pharmacol Ther 2019; 50: 425-34.

26. Wagner AK, Soumerai SB, Zhang F, et al. Segmented regression analysis of interrupted time series studies in medication use research. J Clin Pharm Ther 2002; 27: 299-309.

27. Scottish Medicines Consortium. Advice on sofosbuvir $400 \mathrm{mg}$ tablet (Sovaldi ${ }^{\circledR}$ ). www.scottishmedicines.org.uk/medicines-advice/sofosbuvir-400mg-tablet-sovaldifullsubmission-96414/ (accessed September 20, 2019)

28. White IR, Royston P. Imputing missing covariate values for the Cox model. Statistics in Medicine 2009; 28: 1982-98.

29. White IR, Royston P, Wood AM. Multiple imputation using chained equations: issues and guidance for practice. Statistics in Medicine 2011; 30: 377-99.

30. Innes H, McAuley A, Alavi M, Valerio H, Goldberg D, Hutchinson SJ. The contribution of health risk behaviors to excess mortality in American adults with chronic hepatitis C: A population cohort-study. Hepatology 2018; 67: 97-107.

31. Flemming JA, Kim WR, Brosgart CL, Terrault NA. Reduction in liver transplant wait-listing in the era of direct-acting antiviral therapy. Hepatology 2017; 65: 804-12.

32. Belli LS, Perricone G, Adam R, et al. Impact of DAAs on liver transplantation: Major effects on the evolution of indications and results. An ELITA study based on the ELTR registry. $J$ Hepatol 2018; 69: 810-7.

33. Alavi M, Law MG, Valerio H, et al. Declining hepatitis C virus-related liver disease burden in the direct-acting antiviral therapy era in New South Wales, Australia. J Hepatol 2019; 71: 281-8.

34. Carrat F, Fontaine H, Dorival C, et al. Clinical outcomes in patients with chronic hepatitis C after direct-acting antiviral treatment: a prospective cohort study. Lancet 2019; 393: 1453-64.

35. Smith S, Harmanci H, Hutin Y, et al. Global progress on the elimination of viral hepatitis as a major public health threat: an analysis of WHO Member State responses 2017. JHEP Reports 2019; 1: 81-89.

36. Palmateer NE, Hutchinson SJ, McLeod A, Codere G, Goldberg DJ. Comparison of deaths related to Hepatitis C and AIDS in Scotland. J Viral Hepat 2007; 14: 870-4.

37. Aspinall EJ, Hutchinson SJ, Janjua NZ, et al. Trends in mortality after diagnosis of hepatitis C virus infection: an international comparison and implications for monitoring the population impact of treatment. J Hepatol 2015 Feb; 62: 269-77.

38. Hickman M, Dillon JF, Elliott L, et al. Evaluating the population impact of hepatitis C direct acting antiviral treatment as prevention for people who inject drugs (EPIToPe) - a natural experiment (protocol). BMJ Open 2019; 9: e029538.

39. Palmateer N, McAuley A, Dillon J, et al. Reduction in the population prevalence of chronic HCV among people who inject drugs associated with major scale-up of direct-acting antiviral therapy in community drug services: real world data. 8th International Conference on Hepatitis Care in Substance Users. Montreal: 11th September 2019.

40. Goldberg D, Hutchinson S, Innes H, Dillon J, on behalf of Scotland's Hepatitis C Stakeholders. Scotland's Hepatitis C Action Plan: achievements of the first decade and proposals for a Scottish Government strategy (2019) for the elimination of both infection and disease. Taking advantage of outstanding new therapies. Health Protection Scotland, July 2019. hpspubsrepo.blob.core.windows.net/hps-website/nss/2840/documents/1 HCV-EliminationScotland-2019-07-31.pdf (accessed September 20, 2019)

41. Scottish Government. Effective elimination of hepatitis C by 2024. Scottish Government, July 2019. www.gov.scot/news/eliminating-hepatitis-c/ (accessed September 20, 2019) 


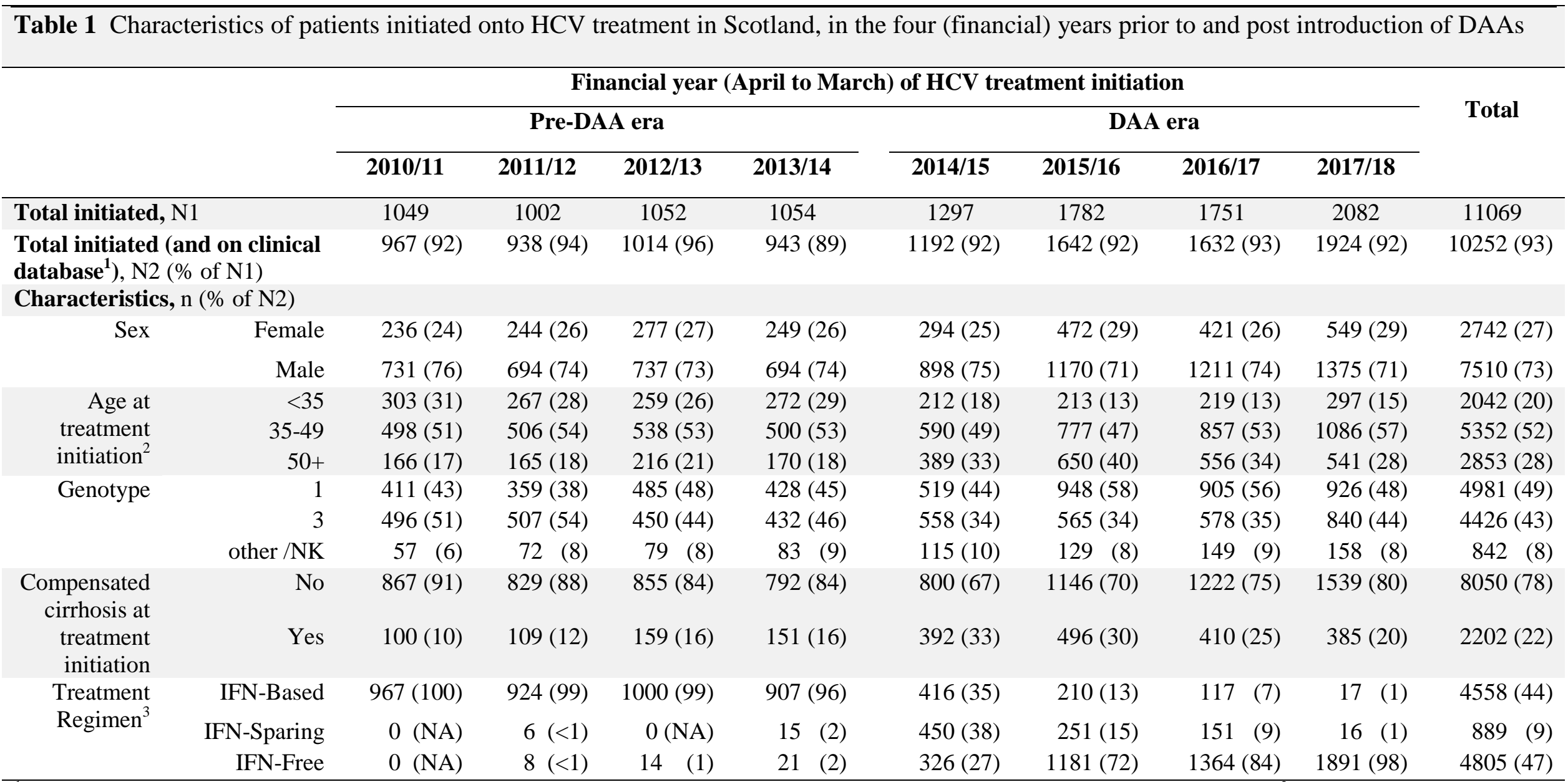

${ }^{1}$ Data collated from 17/18 HCV treatment clinics in Scotland; aggregate data for the remaining clinic sourced for the Scotland total. ${ }^{2}$ Data missing for 5 patients. ${ }^{3}$ IFN-Based includes pegylated interferon + ribavirin \pm telaprevir/boceprevir; IFN-Sparing includes pegylated interferon + ribavirin + sofosbuvir; pegylated interferon + ribavirin + simeprevir; IFN-Free includes any treatment regimen not containing interferon.

HCV, hepatitis C virus; NK, not known; NA, not applicable; IFN, interferon. 
Table 2 a) Characteristics of patients newly presenting with HCV-related decompensated cirrhosis (DC) in the pre-DAA and DAA eras, and b) Results of segmented Poisson regression analyses modelling the trend in the (6-monthly) number of new presentations with HCV-related DC between the pre-DAA and DAA eras (overall and stratified according to patient characteristics).

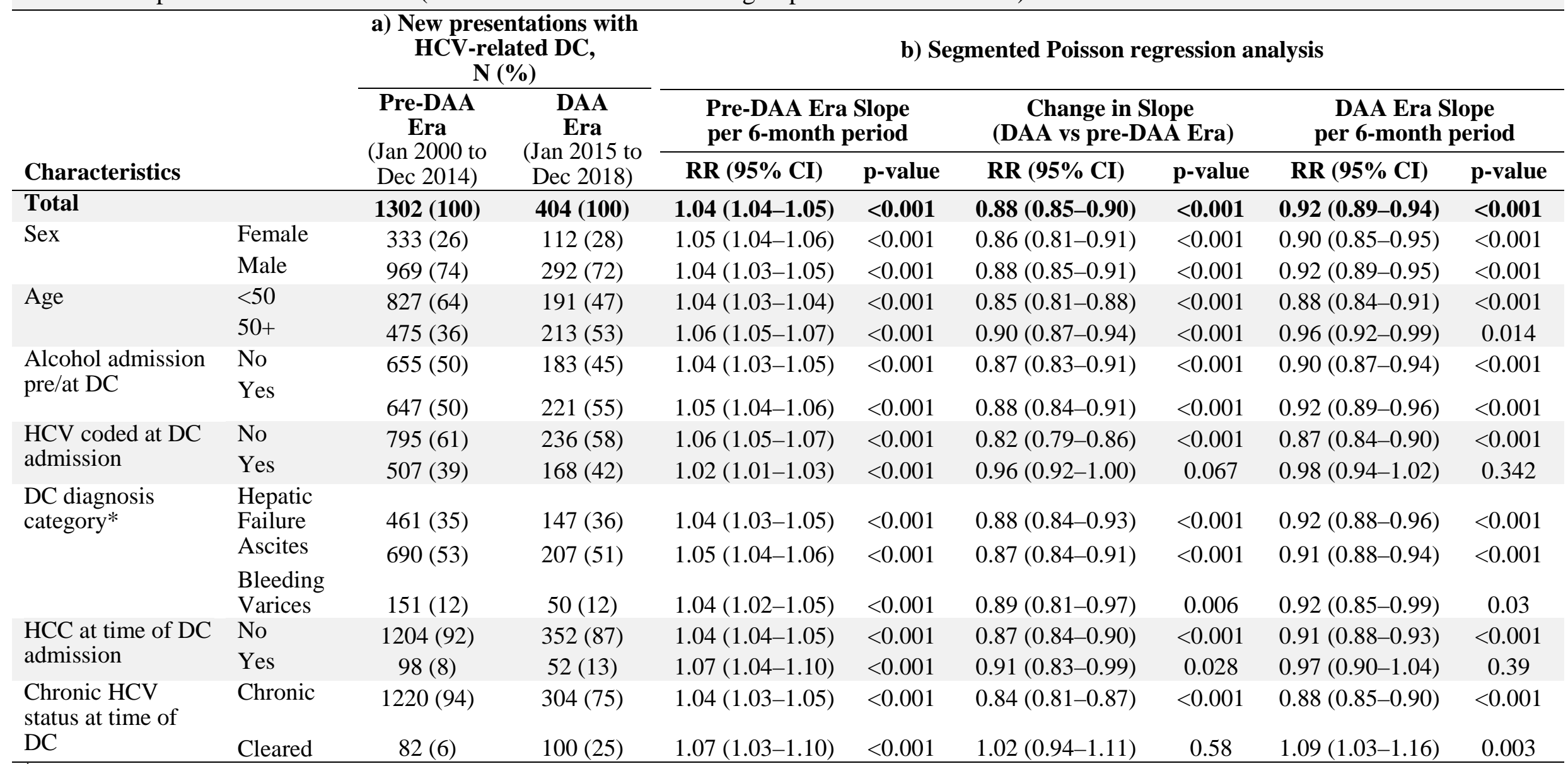

* DC diagnosis category was defined using a hierarchy of ICD-10 codes, ranked in order of hepatic failure, ascites and then bleeding varices.

RR, relative risk; CI, confidence interval; DAA, direct-acting antiviral; DC, decompensated cirrhosis; HCV, hepatitis C virus; HCC, hepatocellular carcinoma; SVR, sustained viral response. 
Table 3 a) Characteristics of chronic HCV infected patients diagnosed with compensated cirrhosis in Scotland between 1 January 2011 and 31 March 2018, b) number (and rate) of and c) hazard ratio for new presentation with HCV-related DC among this cirrhotic population according to DAA era of cirrhosis diagnosis and other covariates.

\begin{tabular}{|c|c|c|c|c|c|c|c|}
\hline & & \multicolumn{2}{|c|}{ a) Cirrhosis population } & \multicolumn{2}{|c|}{$\begin{array}{l}\text { b) New presentation with } \\
\text { HCV-related DC }\end{array}$} & \multicolumn{2}{|c|}{$\begin{array}{c}\text { c) Hazard ratio (HR) for } \\
\text { new presentation with } \\
\text { HCV-related DC }(95 \% \mathrm{CI})\end{array}$} \\
\hline & & N (\%) & Person-years & $\mathrm{n}$ & $\begin{array}{l}\text { Rate (per } 100 \\
\text { person-years) }\end{array}$ & Unadjusted & Adjusted \\
\hline All patients & & $2249(100)$ & 6738 & 219 & $3 \cdot 25$ & - & - \\
\hline Diagnosed with & Pre-DAA era & 1015 (45) & 4529 & 159 & $3 \cdot 51$ & $1 \cdot 00$ & $1 \cdot 00$ \\
\hline cirrhosis & DAA era & $1234(55)$ & 2209 & 60 & $2 \cdot 72$ & $0 \cdot 59(0 \cdot 43-0 \cdot 80)$ & $0.55(0 \cdot 40-0 \cdot 76)$ \\
\hline \multirow[t]{2}{*}{ Sex } & Female & $584(26)$ & 1769 & 59 & $3 \cdot 33$ & $1 \cdot 00$ & $1 \cdot 00$ \\
\hline & Male & $1665(74)$ & 4969 & 160 & $3 \cdot 22$ & $0.96(0 \cdot 71-1 \cdot 30)$ & $0.93(0.68-1.27)$ \\
\hline Age at cirrhosis & $<50$ & $1402(62)$ & 4331 & 122 & $2 \cdot 82$ & $1 \cdot 00$ & $1 \cdot 00$ \\
\hline diagnosis (years) & $50+$ & 847(38) & 2407 & 97 & $4 \cdot 03$ & $1 \cdot 40(1 \cdot 07-1.83)$ & $1 \cdot 61(1 \cdot 19-2 \cdot 20)$ \\
\hline Past/current risk & PWID & $1448(64)$ & 4304 & 129 & $3 \cdot 00$ & $1 \cdot 00$ & $1 \cdot 00$ \\
\hline \multirow[t]{2}{*}{ group } & Non-PWID & 348 (15) & 1166 & 36 & $3 \cdot 09$ & $1 \cdot 08(0 \cdot 77-1 \cdot 50)$ & $0.96(0.63-1.46)$ \\
\hline & Not known* & $453(20)$ & 1268 & 54 & $4 \cdot 26$ & - & - \\
\hline Alcohol & $>50$ units/wk & $743(33)$ & 2207 & 102 & $4 \cdot 62$ & $1 \cdot 78(1 \cdot 37-2 \cdot 32)$ & $1 \cdot 67(1 \cdot 26-2 \cdot 20)$ \\
\hline consumption history & $\begin{array}{r}\leq 50 \text { units/wk (and } \\
\text { not known) }\end{array}$ & $1506(67)$ & 4531 & 117 & $2 \cdot 58$ & $1 \cdot 00$ & $1 \cdot 00$ \\
\hline \multirow[t]{3}{*}{ Genotype } & G1 & $930(41)$ & 2752 & 92 & $3 \cdot 34$ & $1 \cdot 09(0 \cdot 82-1 \cdot 45)$ & $1 \cdot 03(0 \cdot 77-1 \cdot 38)$ \\
\hline & G3 & $1041(46)$ & 3243 & 98 & $3 \cdot 02$ & $1 \cdot 00$ & $1 \cdot 00$ \\
\hline & Other/NK & $278(12)$ & 743 & 29 & $3 \cdot 90$ & $1 \cdot 27(0 \cdot 84-1 \cdot 92)$ & $1 \cdot 01(0 \cdot 65-1 \cdot 57)$ \\
\hline \multirow[t]{3}{*}{ Child-Pugh class } & A & $1556(69)$ & 4752 & 131 & $2 \cdot 76$ & $1 \cdot 00$ & $1 \cdot 00$ \\
\hline & B & $62(3)$ & 134 & 14 & $10 \cdot 47$ & $4 \cdot 03(2 \cdot 14-7 \cdot 59)$ & $3.95(1.98-1 \cdot 57)$ \\
\hline & Not known* & $631(28)$ & 1852 & 74 & $4 \cdot 00$ & - & - \\
\hline \multirow{2}{*}{$\begin{array}{l}\text { Prior antiviral } \\
\text { treatment }\end{array}$} & Naïve & $1876(83)$ & 5177 & 176 & $3 \cdot 40$ & $1 \cdot 00$ & $1 \cdot 00$ \\
\hline & Experienced & $373(17)$ & 1562 & 43 & $2 \cdot 75$ & $0 \cdot 86(0 \cdot 62-1 \cdot 21)$ & $0.75(0.52-1 \cdot 06)$ \\
\hline
\end{tabular}

* Category not used in Cox regression as missing values were imputed (see Methods). 
Figure 1 Number of people initiated on HCV therapy and the estimated number achieving SVR in Scotland by financial year (April to March) of treatment initiation during $2010 / 11$ to $2017 / 18$, for (a) all patients and (b) patients with compensated cirrhosis.

(a) All patients

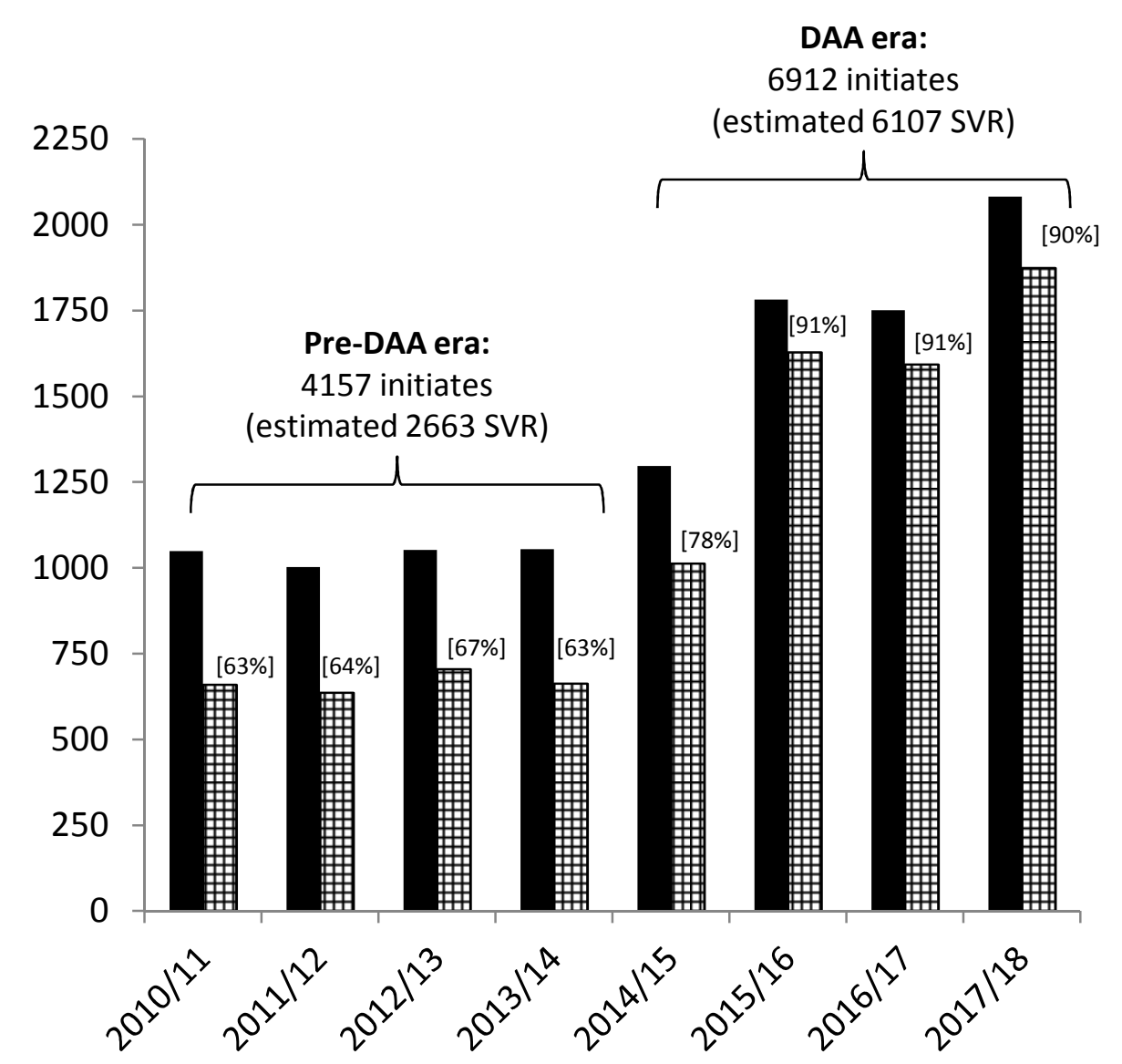

(b) Patients with compensated cirrhosis

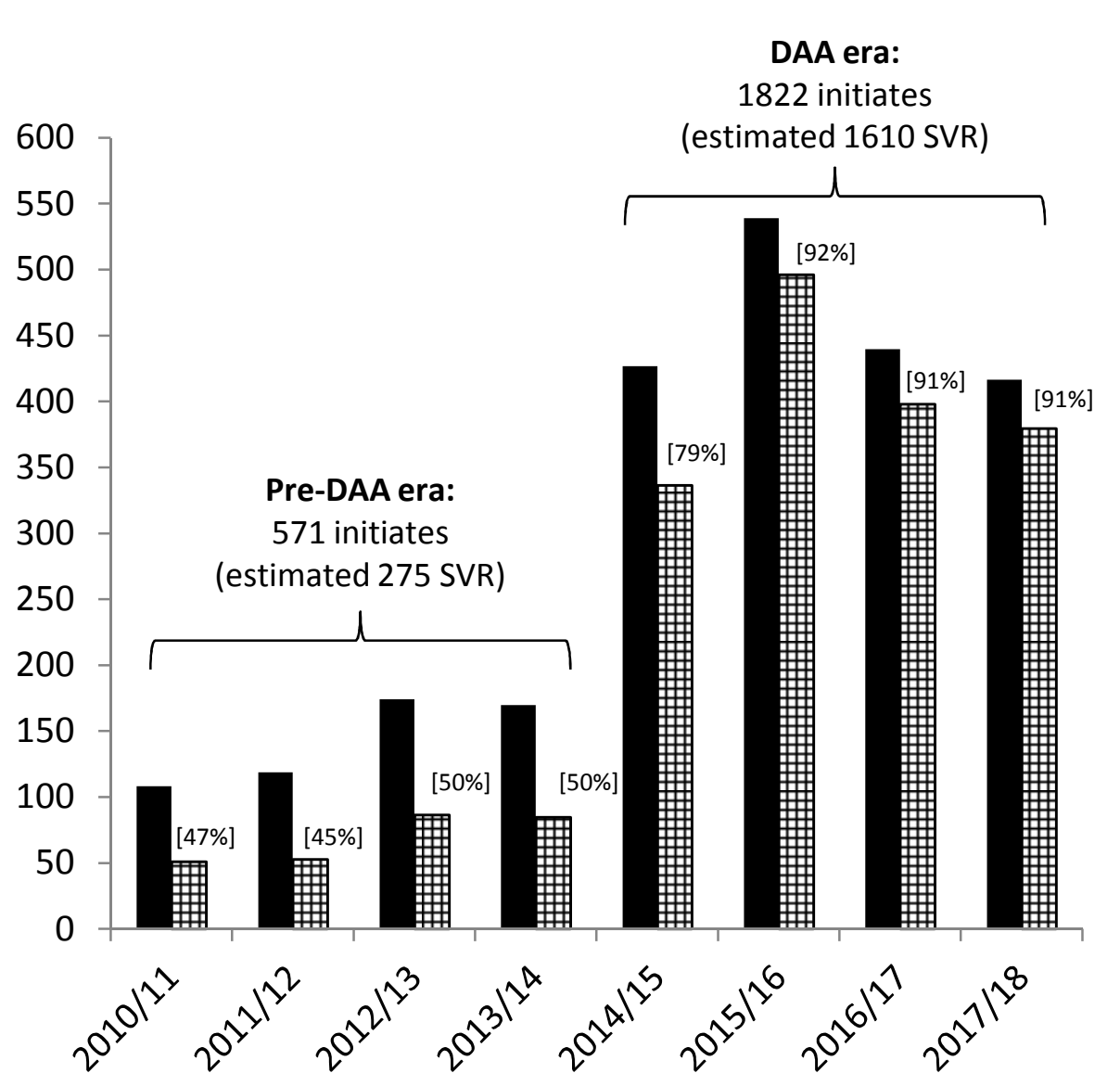

Financial year of treatment initiation 
Figure 2 Observed and predicted number of new presentations for HCV-related decompensated cirrhosis (DC) in Scotland during the pre-DAA (2000-2014) and DAA (2015-2018) eras.

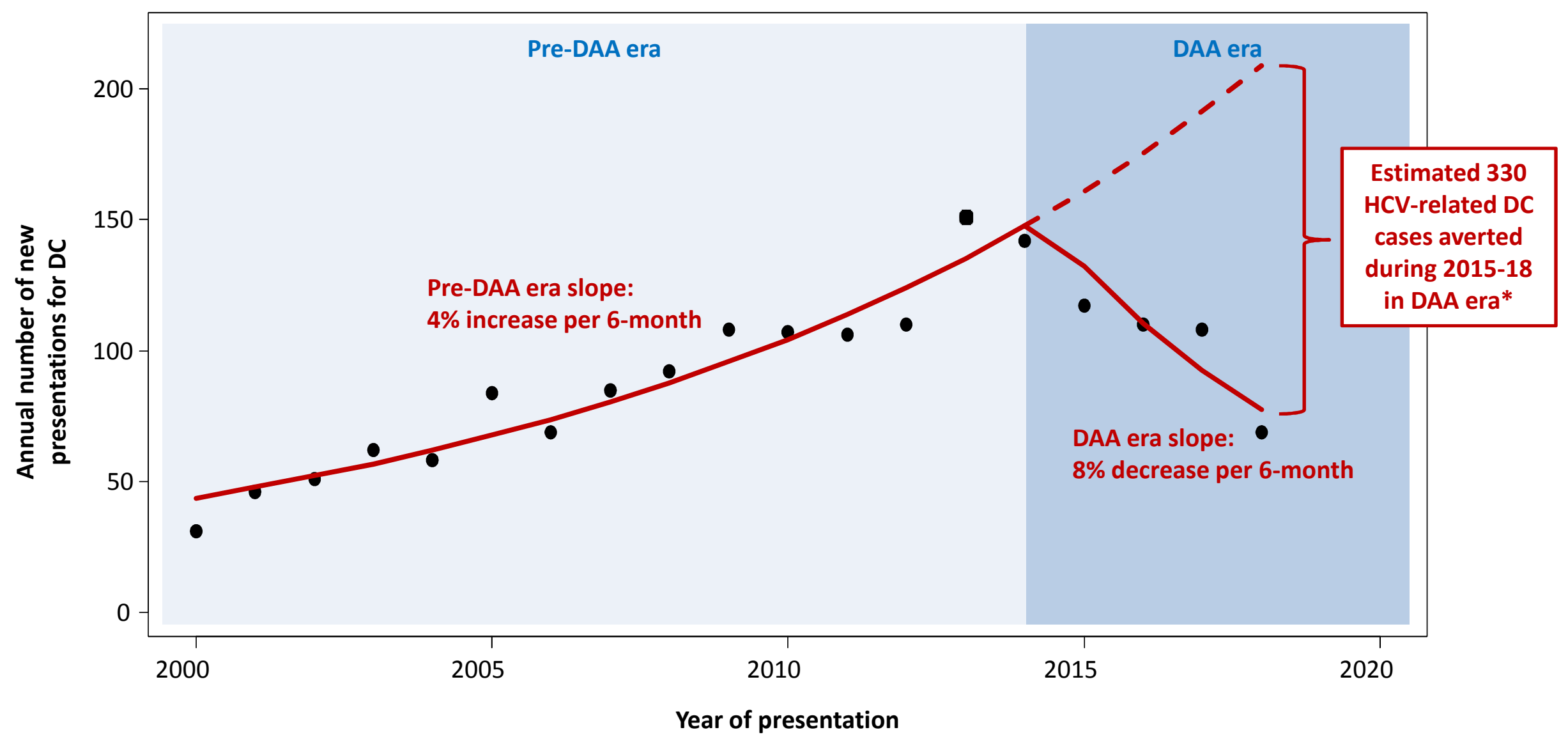
- Observed
Predicted (with DAAs)
---- Predicted (without DAAs) 
Supplementary file 1: Overview of the five national surveillance databases and associated record-linkage involved (indicated by double sided arrows) and analysis/results generated as part of this study.

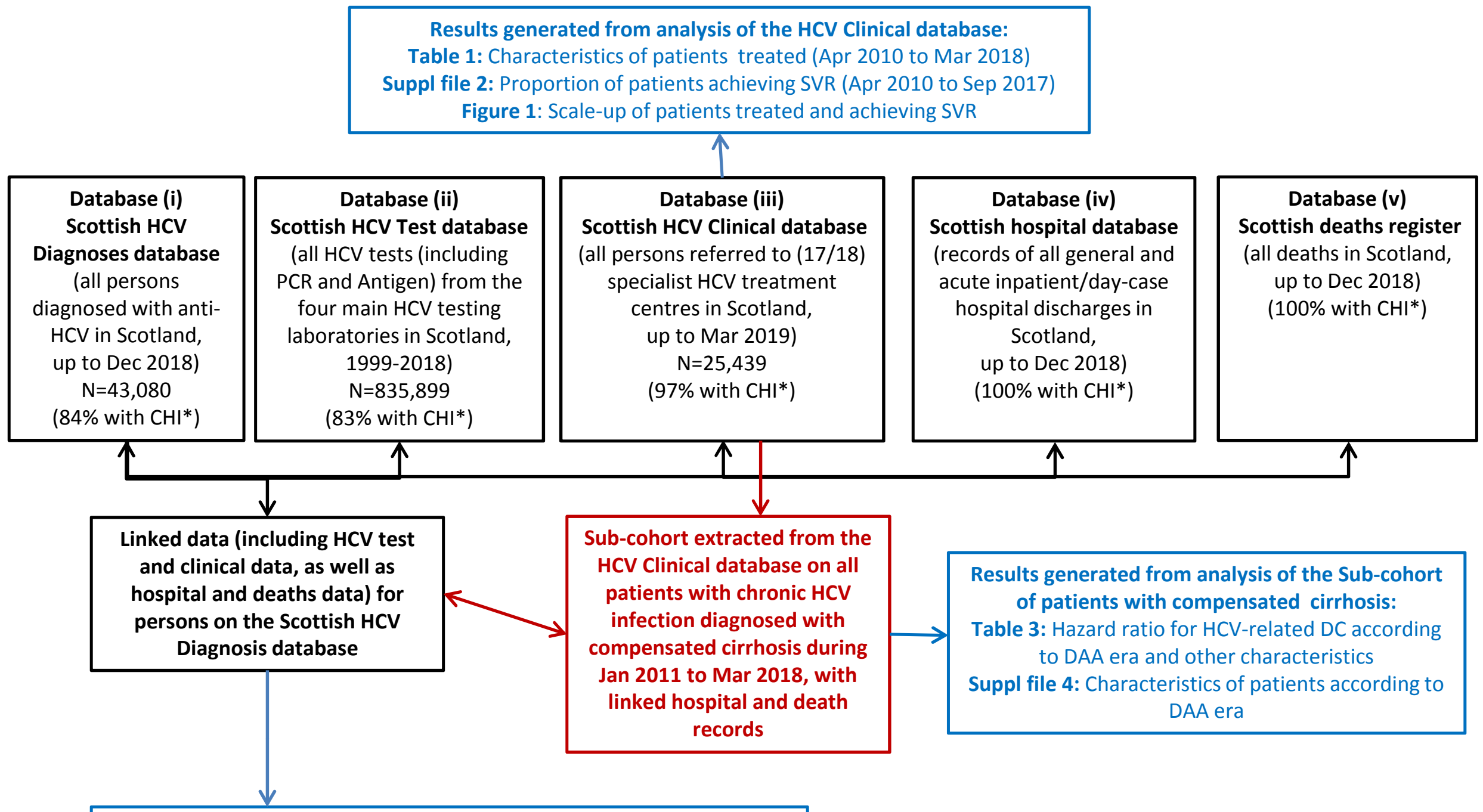

Results generated from analysis of the Linked data :

Table 2: Patients presenting with HCV-related DC (Jan 2000 to Dec 2018)

Figure 2: Trend in presentations with HCV-related DC (2000 to 2018)

Suppl file 3: Sensitivity analysis on definition of HCV-relate d DC

* All databases involved hold the Community Health Index (CHI) number, which is a unique identifier allocated to each patient on first registration with NHS Scotland. Not all records held on the HCV databases will include the CHI number, if not reported from certain settings (e.g. sexual health clinics). As described elsewhere, the $\mathrm{CHI}$ number on the Diagnosis database was used to link and retrieve records from the other databases. ${ }^{7}$ 
Supplementary file 2 Proportion of patients achieving a sustained viral response (SVR) among those initiated on (and with a known response to) HCV treatment in Scotland, by patient characteristics and financial year

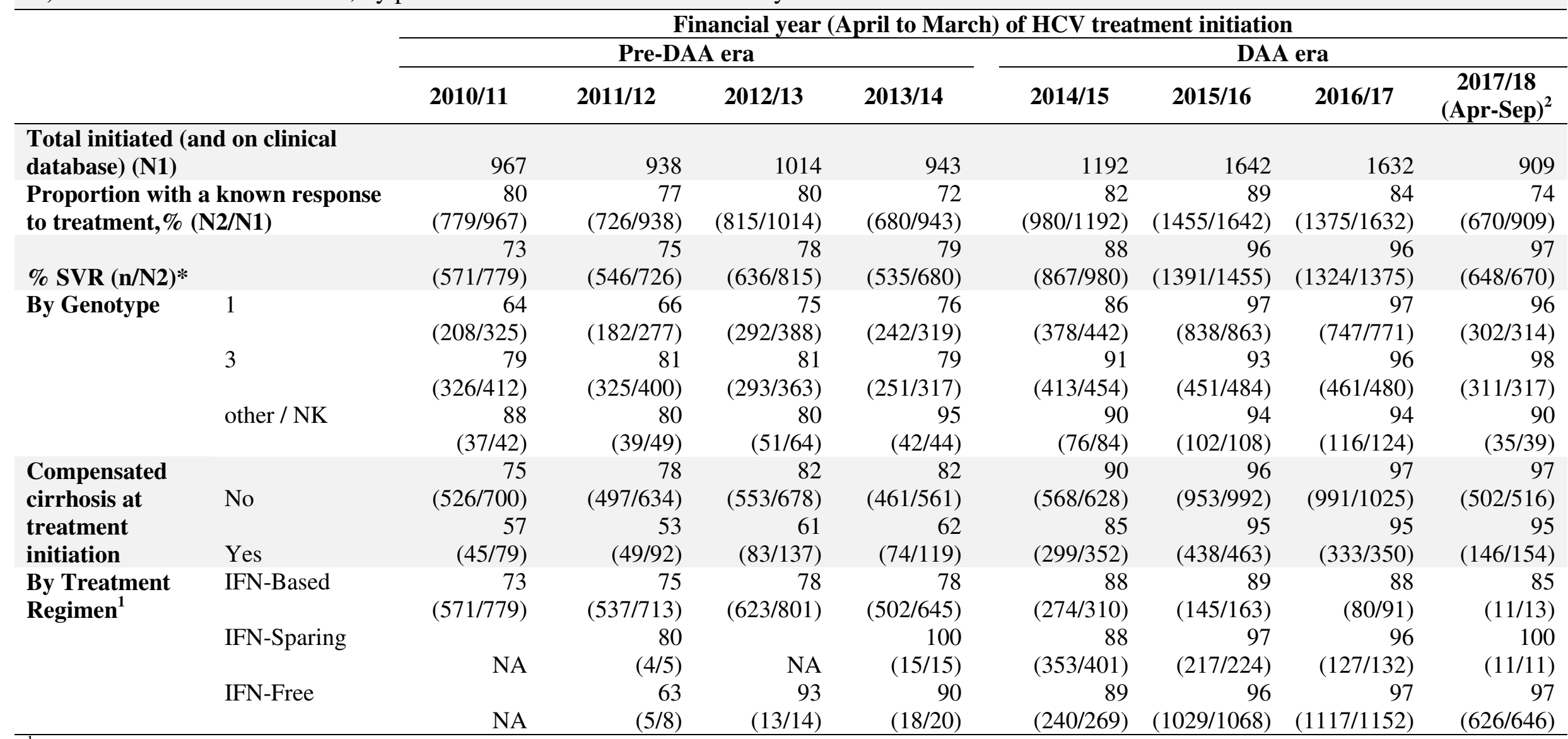

${ }_{1}^{1}$ IFN-Based includes pegylated interferon + ribavirin \pm telaprevir/boceprevir ; IFN-Sparing includes pegylated interferon + ribavirin + sofosbuvir; pegylated interferon + ribavirin + simeprevir ; IFN-Free includes any treatment regimen not containing interferon. ${ }^{2}$ Data relating to those initiated in the first six months of 2017/18 financial year presented, to allow a minimum of six-months follow-up time to capture SVR status on the clinical database. HCV, hepatitis C virus; NK, not known; NA, not applicable; IFN, interferon. 
Supplementary file 3 Results of sensitivity analysis for the segmented regression analysis, considering two broader definitions for the outcome measure of new presentations with HCV-related DC

a) New presentations with
HCV-related DC,
b) Segmented Poisson regression analysis

\begin{tabular}{|c|c|c|c|c|c|c|c|c|c|}
\hline & & \multicolumn{2}{|c|}{ N $(\%)$} & \multicolumn{6}{|c|}{ D) seginemiea roisson regression analysis } \\
\hline & & \multirow{2}{*}{$\begin{array}{c}\text { Pre-DAA } \\
\text { Era } \\
\text { (Jan } 2000 \text { to } \\
\text { Dec 2014) } \\
\end{array}$} & \multirow{2}{*}{$\begin{array}{c}\text { DAA } \\
\text { Era } \\
\text { (Jan 2015 to } \\
\text { Dec 2018) }\end{array}$} & \multicolumn{2}{|c|}{$\begin{array}{l}\text { Pre-DAA Era Slope } \\
\text { per 6-month period }\end{array}$} & \multicolumn{2}{|c|}{$\begin{array}{c}\text { Change in Slope } \\
\text { per 6-month period }\end{array}$} & \multicolumn{2}{|c|}{$\begin{array}{c}\text { DAA Era Slope } \\
\text { per 6-month period }\end{array}$} \\
\hline \multicolumn{2}{|c|}{ Outcome measure } & & & RR $(95 \%$ CI $)$ & p-value & RR $(95 \%$ CI $)$ & p-value & RR $(95 \%$ CI $)$ & p-value \\
\hline i) & $\begin{array}{l}\text { First-time hospital } \\
\text { admission with (or } \\
\text { otherwise death from) } \\
\text { DC, among those with } \\
\text { chronic HCV diagnosis }\end{array}$ & 1415 & 451 & $1.04(1.04-1.05)$ & $<0.001$ & $0.89(0.86-0.91)$ & $<0.001$ & $0.92(0.90-0.95)$ & $<0.001$ \\
\hline ii) & $\begin{array}{l}\text { First-time hospital } \\
\text { admission with DC, } \\
\text { among those with } \\
\text { chronic HCV diagnosis } \\
\text { (including those with } \\
\text { not known chronic } \\
\text { status at diagnosis) }\end{array}$ & 1366 & 412 & $1.04(1.03-1.05)$ & $<0.001$ & $0.88(0.86-0.91)$ & $<0.001$ & $0.92(0.90-0.94)$ & $<0.001$ \\
\hline
\end{tabular}




\begin{tabular}{|c|c|c|c|}
\hline \multicolumn{4}{|c|}{$\begin{array}{l}\text { Supplementary file } 4 \text { Characteristics of chronic HCV infected patients with compensated } \\
\text { cirrhosis in Scotland according to whether diagnosed with cirrhosis during the pre-DAA era } \\
\text { (January } 2011 \text { to May 2014) or in the DAA era (June } 2014 \text { to March 2018) }\end{array}$} \\
\hline & & $\begin{array}{c}\text { Pre-DAA era } \\
\mathrm{N}(\%)\end{array}$ & $\begin{array}{c}\text { DAA era } \\
\mathrm{N}(\%) \\
\end{array}$ \\
\hline Total & & $1015(100)$ & $1234(100)$ \\
\hline \multirow{2}{*}{ Sex } & Female & 275 (27) & 309 (25) \\
\hline & Male & 740 (73) & $925(75)$ \\
\hline \multirow{4}{*}{$\begin{array}{l}\text { Age at cirrhosis } \\
\text { diagnosis (years) } \\
\text { Risk group for HCV } \\
\text { acquisition }\end{array}$} & $<50$ & $661(65)$ & $741(60)$ \\
\hline & $50+$ & 354 (35) & $493(40)$ \\
\hline & PWID & 639 (63) & 809 (66) \\
\hline & Non-PWID/not known & $376(37)$ & $425(34)$ \\
\hline \multirow{2}{*}{$\begin{array}{l}\text { Alcohol consumption } \\
\text { history }\end{array}$} & $>50$ units/wk & $350(34)$ & $393(32)$ \\
\hline & $\begin{array}{r}\leq 50 \text { units/wk (and not } \\
\text { known) }\end{array}$ & $665(66)$ & $841(68)$ \\
\hline \multirow[t]{3}{*}{ Genotype } & G1 & $398(39)$ & $532(43)$ \\
\hline & G3 & 491 (48) & $550(45)$ \\
\hline & Other/NK & $126(12)$ & $152(12)$ \\
\hline \multirow[t]{3}{*}{ Child-Pugh class } & A & $706(70)$ & $850(69)$ \\
\hline & B & $26(3)$ & $36(3)$ \\
\hline & Not known & $283(27)$ & $348(28)$ \\
\hline \multirow{2}{*}{$\begin{array}{l}\text { Prior antiviral } \\
\text { treatment }\end{array}$} & Naïve & $766(75)$ & $1110(90)$ \\
\hline & Experienced & $249(25)$ & 124 (10) \\
\hline
\end{tabular}


Supplementary file 5: Observed number of i) new presentations of HCV-related decompensated cirrhosis (DC), ii) new presentations of HCV-related hepatocellular carcinoma (HCC), and iii) HCV-related liver* deaths (i.e. involving either DC and/or HCC) in Scotland, during the pre-DAA (2000-2014) and DAA (2015-2018) eras.

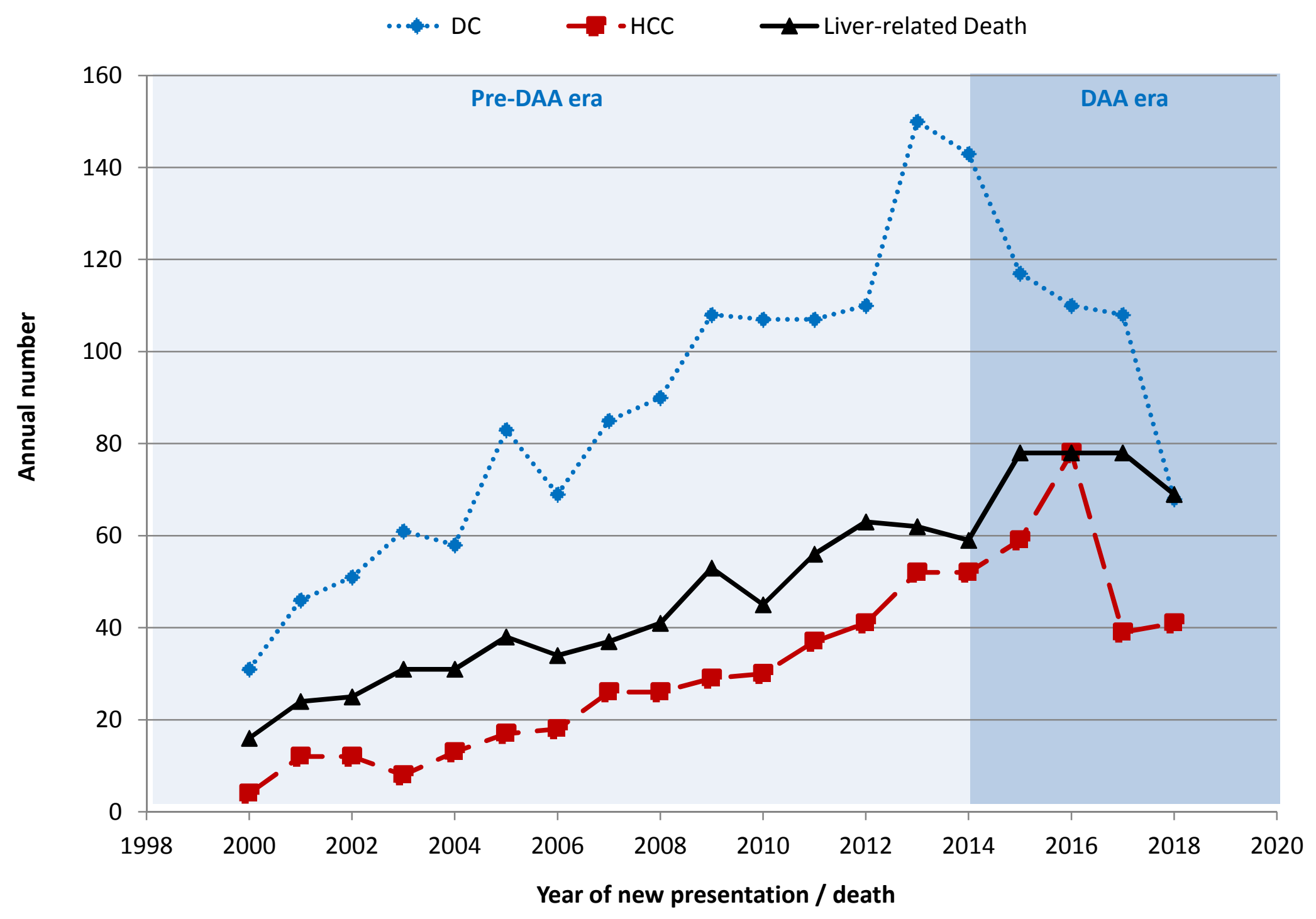

* Liver-related refers to death involving either DC and/or HCC. 